\title{
Infrared photometry of the final flash star V605 Aquilae
}

\author{
K. H. Hinkle, R. R. Joyce, and A. Hedden* \\ National Optical Astronomy Observatory ${ }^{\star \star}, 950$ N. Cherry Avenue, PO Box 26732, Tucson, 85726, USA \\ Received 4 September 2000 / Accepted 24 November 2000

\begin{abstract}
We have obtained infrared images in the $J, H$, and $K$ bands of the field containing the post-AGB final-flash object V605 Aql. V605 Aql is the central star of the planetary nebula A58 but has been incorrectly identified with other stars in this field. In the infrared images V605 Aql appears as a very red star at the position of the A58 center. We present JHK photometry on two dates as well as astrometry of V605 Aql. The infrared light from V605 Aql originates in a thick dust cloud surrounding the star. There is no indication of variability over a three month time period.
\end{abstract}

Key words. stars: evolution - stars: individual: V605 Aql - stars: AGB and post-AGB - planetary nebulae: individual: A58 - infrared: stars

\section{Introduction}

Calculations done in the early 1980's demonstrated that luminous central stars of planetary nebulae could be stars undergoing a final helium shell flash. As reviewed by Iben \& MacDonald (1994) the final helium shell flash brightens a white dwarf to AGB luminosity and results in a sudden change in abundances as the residual hydrogen envelope is consumed in the helium-burning convective shell. This process has been observed in V4334 Sgr (Sakurai's Object), and this star has been identified as a prototypical final helium shell flash object (Asplund et al. 1997). The list of similar objects is small, certainly less than 50, and perhaps as small as a few, depending on whether or not the R CrB stars are included (Clayton \& De Marco 1997).

The only known star with behavior closely mimicing that of Sakurai's star is V605 Aql (Clayton \& De Marco 1997). V605 Aql underwent brightening and fading in 1919-1923 with a light curve similar to that of Sakurai's star in 1996-1999. From 1924 through the early 1980 's the visual faintness of V605 Aql as well as ignorance of its transient evolutionary state resulted in its being largely unobserved (Bidelman 1971; van den Bergh 1971). V605 Aql can be seen on the original Palomar sky survey as a faintly visible star on the red plate. Both V605 Aql and its associated planetary nebula A58 are obvious on a $5 \mathrm{~m}$ Hale image by van den Bergh (1971). However, the

Send offprint requests to: K. H. Hinkle,

e-mail: hinkle@noao.edu

* Based on research conducted at NOAO as part of the Research Experiences for Undergraduates program.

** Operated by the Association of Universities for Research in Astronomy, Inc. under cooperative agreement with the National Science Foundation. connection between the visually faint central star of A58 and V605 Aql, which was lost in 1923, was first made by Seitter (1985). At about the same time IRAS recovered V605 Aql as a bright point source $(19158+0141)$ detected in all four bands, peaking at $41 \mathrm{Jy}$ at $60 \mu \mathrm{m}$. V605 Aql was also observed as a bright and very red source by ISO with flux ranging from $0.5 \mathrm{Jy}$ at $4.5 \mu \mathrm{m}$ to $6.4 \mathrm{Jy}$ at $14.5 \mu \mathrm{m}$ (Kimeswenger et al. 1998).

Following the visual and far-infrared recovery of V605 Aql there has been considerable confusion about the identification of this star. This is reviewed by Kimeswenger et al. (2000) who present the first nearinfrared image of the central star. To summarize, Herbig (1958) produced a finding chart accurate to a few arcseconds and noted a faint star on the Palomar sky survey at this position, but failed to note the presence of the (faint) planetary nebula. van der Veen et al. (1989) and Harrison (1996) later incorrectly identified V605 Aql as a star $35^{\prime \prime}$ from the center of the planetary nebula. As a result these papers reported near-infrared magnitudes far brighter than the correct values and these mistaken values have propagated in the literature. Kimeswenger et al. (2000) correctly identified V605 Aql and identified the nearby field star measured by van der Veen et al. (1989) and Harrison (1996).

Independent of the work of Kimeswenger et al. (2000) we attempted to observe the infrared spectrum of V605 Aql in June 2000. Following the Harrison (1996) paper we observed the incorrect star. We rapidly concluded that this star is not a final flash object and not the central star of A58. In order to identify the correct star we undertook wide field $J H K$ imaging which is reported on here. 


\section{Observations and analysis}

Observations were made on 14 June 2000 UT and 14 September 2000 UT at the KPNO 2.1-m telescope using the facility imager SQIID (Ellis et al. 1992). SQIID utilizes three dichroic beamsplitters to image a field simultaneously at $J, H, K$, and (narrow band) $L$. The June observations were among the first since the SQIID detectors were upgraded from the original low quantum efficiency PtSi arrays to high quantum efficiency Aladdin InSb arrays, increasing the responsivity by factors of $10(J)$ to $50(K)$. The SQIID optics illuminate most of a $512 \times 512$ Aladdin quadrant (but not the entire $1024 \times 1024$ array) with a resultant pixel scale at the 2.1-m of $0 .{ }^{\prime} 69$. Large thermal background due to hot weather at the times of the observations prevented images from being recorded at $L$.

The June observations consisted of five coadded $2 \mathrm{~s}$ observations at five locations (i.e., a total exposure of $50 \mathrm{~s}$ ) centered on V605 Aql and on a calibration star. Each observation yielded three colors, $J, H$, and $K$. The grid of images was a standard five raster set, one with the object in the middle and the other four centered on the corners of a square, with each corner $7^{\prime \prime}$ diagonally offset from the midpoint. Each image was analyzed separately, with the quoted mean and uncertainty being calculated from the five separate measurements. The UKIRT Faint Standards Catalog star FS28 (SA109-71) was used for photometric calibration. The observations were repeated in September with a 4 by 4 grid of 30 coadded 5 s integrations (i.e. a total exposure of $2400 \mathrm{~s}$ ).

IRAF routines were employed in the photometric and astrometric analysis of the images. All images were flat fielded using a dark subtracted flat field derived from sky observations. To produce a sky image the sets of images for each object in each color were combined using a median filter. The sky was then subtracted from each image and photometry was carried out using the "phot" task in the "digiphot" package. Each image was processed separately and the values combined to produce the uncertainty.

V605 Aql appears as a very red star on the images and was too faint to detect on individual June $J$ images. To measure the position of V605 Aql astrometry was carried out on the $H$ and $K$ images, using the "tfinder" and "tastrom" tasks in the IRAF finder package. "tfinder" uses the Hubble Guide Star catalogue for the reference star coordinates. To compare the Guide Star coordinates with USNO A2.0 coordinates we solved for the USNO A2.0 stars in our images. There does appear to be a small (less than $1^{\prime \prime}$ ) systematic offset between the two coordinate systems, most likely reflecting the limiting precision of the Guide Star coordinates.

\section{Discussion}

Individual $J, H$, and $K$ images were shifted and averaged to yield the combined frames shown in Fig. 1. V605 Aql is not visible on some individual $J$ images, but can be seen in the combined images. In June $J=18.7 \pm 0.3$ and in

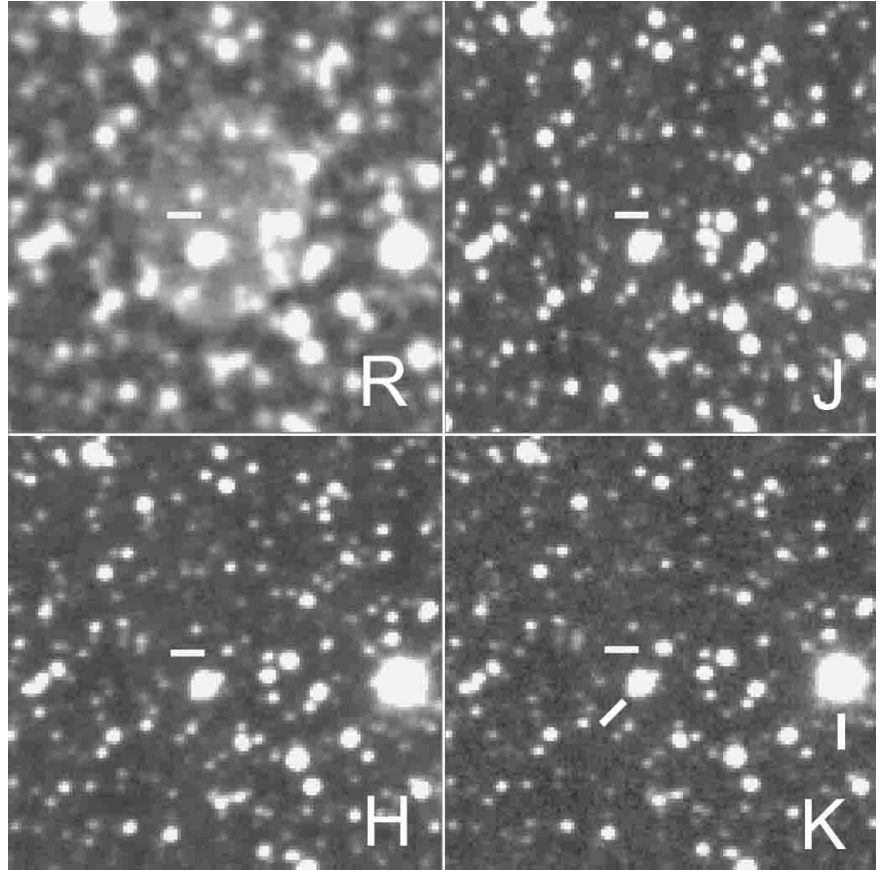

Fig. 1. Images of the V605 Aql field. Only the $88^{\prime \prime} \times 88^{\prime \prime}$ section centered on V605 Aql is shown. The $R$ band image is from the DSS2. The $J H K$ images are the September 2000 SQIID data. V605 Aql is to the right of the horizontal line near the center of each image. In the $R$ image both the planetary nebula A58 and the central star V605 Aql can be seen. In the $K$ image we have marked the Herbig offset star (just above and to the right of the diagonal line just below the center of the image) and the van der Veen/Harrison star (bright star just above vertical line at right edge of image)

September $J=18.1 \pm 0.1$. The average $H$ and $K$ photometry from the individual images yield $H=16.78 \pm 0.15$ and $K=14.67 \pm 0.04$ for the June images and $H=16.43 \pm 0.05$ and $K=14.63 \pm 0.03$ for the September images. The formal uncertainty is the standard deviation of the individual measurements. The $J-H-K$ color temperature of V605 Aql is $\sim 1000 \mathrm{~K}$ but the three points are not perfectly defined by a blackbody. The June and September magnitudes agree at about the size of the uncertainty and no variation in the magnitude of V605 Aql is indicated. The observed flux is significantly less (by $\sim 1.5 \mathrm{mag}$ at $J ; 3.7$ mag at $K$ ) than that predicted by Kimeswenger et al. (2000).

Astrometry of V605 Aql in the June $H$ and $K$ images yields the position

$19^{\mathrm{h}} 18^{\mathrm{m}} 20.5 \pm 0.6+1^{\circ} 46^{\prime} 58^{\prime \prime} .7 \pm 1^{\prime \prime} .0(2000)$

relative to the HST Guide Star reference frame. As noted above, the HST and USNO A2 reference frames differ in this field by nearly $1^{\prime \prime}$. A shift between TYCHO2 and USNO A2 coordinate systems of a similar magnitude is mentioned by Kimeswenger et al. (2000). V605 Aql is visible on both the red and blue Digital Sky Survey 2 
(DSS2) images ${ }^{1}$. From the DSS2 red image we find the position for V605 Aql to be

$19^{\mathrm{h}} 18^{\mathrm{m}} 20 \mathrm{~s} 56 \pm 0$ s $17+1^{\circ} 46^{\prime} 58^{\prime \prime} \cdot 8 \pm 0^{\prime \prime} \cdot 3(2000)$

using the HST guide star reference frame. The infrared and DSS2 positions agree to within the uncertainty. The coordinates agree nicely with those measured from the Palomar Sky Survey by Pollacco et al. (1992). However, the coordinates differ slightly from those of Kimeswenger et al. (2000) which were apparently measured from the DSS2. The disagreement in RA is within the uncertainty but the disagreement in declination is more than $2^{\prime \prime}$.

We confirm the Kimeswenger et al. (2000) identification of the star measured by van der Veen et al. (1989) and Harrison (1996). We measure magnitudes of $J=10.16$, $H=9.48, K=9.07$ with formal uncertainties of better than 0.01 magnitude. It must be emphasized that since the star measured by van der Veen et al. (1989) and Harrison (1996) was not V605 Aql, near infrared magnitudes reported for $\mathrm{V} 605 \mathrm{Aql}$ up to now in the literature are incorrect and must be disregarded. We have also measured, using the DSS2, the position of the van der Veen/Harrison star, $19^{\mathrm{h}} 18^{\mathrm{m}} 18^{\mathrm{s}} .16+1^{\circ} 46^{\prime} 51^{\prime \prime} \cdot 9$, and the 17th magnitude offset star mentioned by Herbig, $19^{\mathrm{h}} 18^{\mathrm{m}} 20.81+1^{\circ} 46^{\prime} 51^{\prime \prime} .8$. The infrared magnitudes of the Herbig star are $J=12.77, H=11.99, K=11.82$. Observers may find these field stars useful for setting up on V605 Aql.

The spatial resolution of the infrared images reported in this paper is relatively coarse, $\sim 1$ ". 4 . While these images are useful for measuring the overall infrared properties of V605 Aql, it has been pointed out by Pollacco et al. (1992) that the expanding material resulting from the final flash of V605 Aql should now have a diameter on the order of $1^{\prime \prime}$. Clayton \& De Marco (1997) and Guerrero \& Manchado (1996) have marginally resolved this shell, referred to as the central knot of A58, using optical emission lines. The infrared radiation originates in optically thick dust in the central knot (Clayton \& De Marco 1997) and comparison of images of the dust with images of the emission lines would be revealing of the geometry.

Acknowledgements. We thank Dr. K. Michael Merrill for assisting with SQIID. We are indebted to Dr. Glenn Tiede for obtaining the observation on 14 September. We acknowledge Dr. George Jacoby for useful discussions and thank him for providing an observation of the V605 Aql field and for noting the image of V605 Aql on the blue DSS2. We thank the referees, Dr. F. Kerber and Dr. C. Waelkens, for comments that improved this paper. This research made use of the SIMBAD database, operated by CDS in Strasbourg, France, and NASA's Astrophysics Data System Abstract Service.

\section{References}

Asplund, M., Gustafsson, B., Lambert, D. L., \& Kameswara Rao, N. 1997, A\&A, 321, L17

Bidelman, W. P. 1971, ApJ, 165, L7

Clayton, G. C., \& De Marco, O. 1997, AJ, 114, 2679

Ellis, T., Drake, R., Fowler, A. M., et al. 1992, Proc. SPIE, 1765,94

Guerrero, M. A., \& Manchado, A. 1996, ApJ, 472, 711

Harrison, T. E. 1996, PASP, 108, 1112

Herbig, G. H. 1958, PASP, 70, 605

Iben, I. Jr., \& MacDonald, J. 1995, The Born Again AGB Phenomenon, in ed. D. Koester, \& K. Werner, White Dwarfs, Springer Lecture Notes in Physics, 443, 48

Kimeswenger, S., Kerber, F., \& Weinberger, R. 1998, MNRAS, 296,614

Kimeswenger, S., Koller, J., \& Schmeja, S. 2000, A\&A, 360, 699

Pollacco, D. L., Lawson, W. A., Clegg, R. E. S., \& Hill, P. W. 1992, MNRAS, 257, 33p

Seitter, W. C. 1985, Mitt. Astron. Ges., 63, 181

Seitter, W. C. 1987, Messenger, 50, 14

van den Bergh, S. 1971, PASP, 83, 819

van der Veen, W. E. C. J., Habing, H. J., \& Geballe, T. R. 1989, A\&A, 226, 108

${ }^{1}$ Clayton \& De Marco (1997) note that the stellar visual flux may be largely contributed by Wolf-Rayet lines with a stellar visual magnitude $\sim 23$ in the continuum (Seitter 1987). This appears to be supported by a CCD image taken at WIYN by George Jacoby. The image was taken at a wavelength between emission lines and V605 Aql is not visible. The limiting magnitude of the exposure is $\sim 23$ but the image unfortunately lacks photometric calibration. 